\title{
Combating Oxidative Stress and Inflammation in COVID-19 by Molecular Hydrogen Therapy: Mechanisms and Perspectives
}

\author{
Duried Alwazeer $\mathbb{D}^{1},{ }^{1,2,3}$ Franky Fuh-Ching Liu $\mathbb{D}^{\mathrm{D}},{ }^{4}$ Xiao Yu Wu ${ }^{\mathrm{D}}{ }^{4}$ \\ and Tyler W. LeBaron $\mathbb{1}^{5,6,7}$ \\ ${ }^{1}$ Department of Nutrition and Dietetics, Faculty of Health Sciences, Igdir University, 76000 Igdir, Turkey \\ ${ }^{2}$ Research Center for Redox Applications in Foods (RCRAF), Igdir University, 76000 Igdir, Turkey \\ ${ }^{3}$ Innovative Food Technologies Development, Application, and Research Center, Igdir University, 76000 Igdir, Turkey \\ ${ }^{4}$ Advanced Pharmaceutics and Drug Delivery Laboratory, Leslie L. Dan Faculty of Pharmacy, University of Toronto, Toronto, ON, \\ Canada M5S 3M2 \\ ${ }^{5}$ Center of Experimental Medicine, Institute for Heart Research, Slovak Academy of Sciences, Bratislava, Slovakia \\ ${ }^{6}$ Molecular Hydrogen Institute, Enoch, Utah, USA \\ ${ }^{7}$ Department of Kinesiology and Outdoor Recreation, Southern Utah University, Cedar City, 84720 Utah, USA
}

Correspondence should be addressed to Duried Alwazeer; alwazeerd@gmail.com and Tyler W. LeBaron; sci_ty7@yahoo.com

Received 21 January 2021; Accepted 20 September 2021; Published 4 October 2021

Academic Editor: Md Saquib Hasnain

Copyright (c) 2021 Duried Alwazeer et al. This is an open access article distributed under the Creative Commons Attribution License, which permits unrestricted use, distribution, and reproduction in any medium, provided the original work is properly cited.

\begin{abstract}
COVID-19 is a widespread global pandemic with nearly 185 million confirmed cases and about four million deaths. It is caused by an infection with the severe acute respiratory syndrome coronavirus-2 (SARS-CoV-2), which primarily affects the alveolar type II pneumocytes. The infection induces pathological responses including increased inflammation, oxidative stress, and apoptosis. This situation results in impaired gas exchange, hypoxia, and other sequelae that lead to multisystem organ failure and death. As summarized in this article, many interventions and therapeutics have been proposed and investigated to combat the viral infection-induced inflammation and oxidative stress that contributes to the etiology and pathogenesis of COVID-19. However, these methods have not significantly improved treatment outcomes. This may partly be attributable to their inability at restoring redox and inflammatory homeostasis, for which molecular hydrogen $\left(\mathrm{H}_{2}\right)$, an emerging novel medical gas, may complement. Herein, we systematically review the antioxidative, anti-inflammatory, and antiapoptotic mechanisms of $\mathrm{H}_{2}$. Its small molecular size and nonpolarity allow $\mathrm{H}_{2}$ to rapidly diffuse through cell membranes and penetrate cellular organelles. $\mathrm{H}_{2}$ has been demonstrated to suppress NF- $\kappa$ B inflammatory signaling and induce the Nrf2/Keap1 antioxidant pathway, as well as to improve mitochondrial function and enhance cellular bioenergetics. Many preclinical and clinical studies have demonstrated the beneficial effects of $\mathrm{H}_{2}$ in varying diseases, including COVID-19. However, the exact mechanisms, primary modes of action, and its true clinical effects remain to be delineated and verified. Accordingly, additional mechanistic and clinical research into this novel medical gas to combat COVID-19 complications is warranted.
\end{abstract}

\section{Introduction: Clinical Challenges and Dilemma of COVID-19 Treatments}

COVID-19 (initially named 2019 novel coronavirus, or 2019-nCoV disease, after the first reported outbreak in 2019) has become the most widely spread global pandemic in the past century [1]. It has affected 189 countries and regions with nearly 185 million confirmed cases and about four million reported deaths worldwide as of current statistics [2]. The novel coronavirus responsible for this disease was named by the World Health Organization the Severe Acute Respiratory Syndrome Coronavirus-2 (SARS-CoV-2) for its genetic similarity to the coronavirus that caused the SARS outbreak in 2003 (SARS-CoV) [1]. While not 


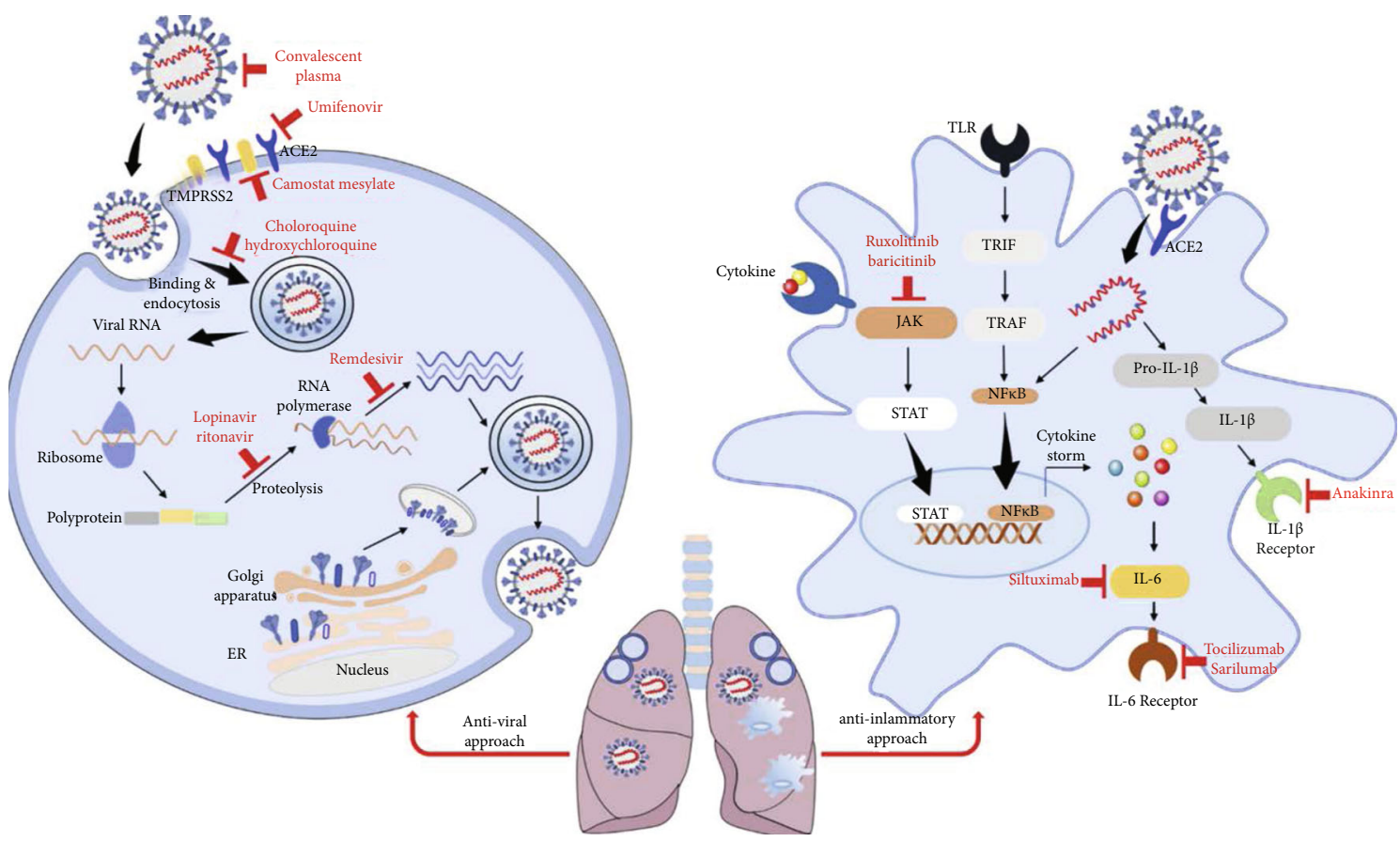

FIGURE 1: Illustration of various pharmacological therapies proposed and investigated to treat COVID-19 patients classified as two categories: antiviral approach and anti-inflammatory approach. The antiviral approach includes the use of agents that block viral binding, entry, fusion, RNA duplication, viral assembly, or exocytosis. The anti-inflammatory approach includes the application of agents that inhibit various inflammatory pathways, reduce cytokine production, and block cytokine receptors. Reproduced with permission from the publisher [4].

ominously fatal as contracting SARS-CoV, COVID-19's mild symptoms and asymptomatic transmission, coupled with long incubation time and long fomite survival time of the virus have complicated epidemic control globally.

Most COVID-19 cases manifest as a respiratory illness with vague symptomatology, starting with a fever, dry cough, and fatigue, followed by shortness of breath with worsening disease. About $80 \%$ of infected people may recover from the illness without hospitalization; yet, the remainder $(20 \%)$ progress to pneumonia and severe acute respiratory distress syndrome (ARDS) [3]. An estimated $5 \%$ of patients require treatment in an intensive care unit (ICU), requiring ventilation for oxygenation and intubation to support life [3]. Of these critically ill patients in ICU, approximately half eventually die of infection-associated complications, typically following multiple organ injury and failure [3]. COVID-19 complications have been correlated to underlying medical conditions, particularly older adults with hypertension, diabetes, and/or other cardiovascular diseases.

In contrast, cytokine storms, caused by an overactive host immune system to any infection, are most responsible for mortality in young and middle-aged patients without medical histories. Current treatment modalities, including antiviral, anti-inflammatory (Figure 1), antimalarial, immunoregulatory therapeutics, ventilation, and extracorporeal membrane oxygenation (ECMO), attempt to mitigate the sequelae caused by infection (Table 1), but they cannot fully address the upstream factors that lead to "cytokine storms," which contribute to multiple organ failure and sudden deaths.
The coronavirus appears to exploit angiotensinconverting enzyme II (ACE2) as a receptor for cell binding and entry. ACE2 is expressed abundantly on epithelial cells in certain mucosal tissues [6]. Of note, the oral and nasal mucosa, eyes, and upper respiratory tract are the primary anatomical inoculation points for viruses that are mainly transmitted via aerosol droplets, propagated from human carriers in close proximity. The infection progresses to lower airways, particularly to alveolar epithelial cells that are susceptible to viral entry. When this occurs, alveolar macrophages and infiltrated immune cells are activated, which then increases oxygen consumption exacerbating alveolar hypoxia [7]. Activated alveolar macrophages also release proinflammatory cytokines within alveoli and pulmonary microvessels, which then enter the systemic circulation. Because injured lungs cannot effectively deliver oxygen or eliminate carbon dioxide from the bloodstream, systemic hypoxia (namely, hypoxemia) and hypercapnia develop. Both alveolar hypoxia and hypoxemia further induce inflammatory cascades, leading to the production of excess reactive oxygen species (ROS) and activation of hypoxia-inducible factors (HIF-1 $\alpha$ ), nuclear factor-kappa-light-chain-enhancer of activated B cells (NF- $\kappa \mathrm{B})$, and proinflammatory cytokines [7]. Thus, oxygen inhalation and anti-inflammatory therapy are considered essential for severe COVID-19, in addition to other potentially useful therapies.

However, in severe COVID-19 pneumonia, inflammation of the respiratory tract and exudation of viscous mucus in bronchioles and alveoli make oxygenation of blood inefficient. Despite high-speed oxygen ventilation, oxygen cannot 
TABLE 1: Selected treatments investigated in clinical trials for COVID-19.*.

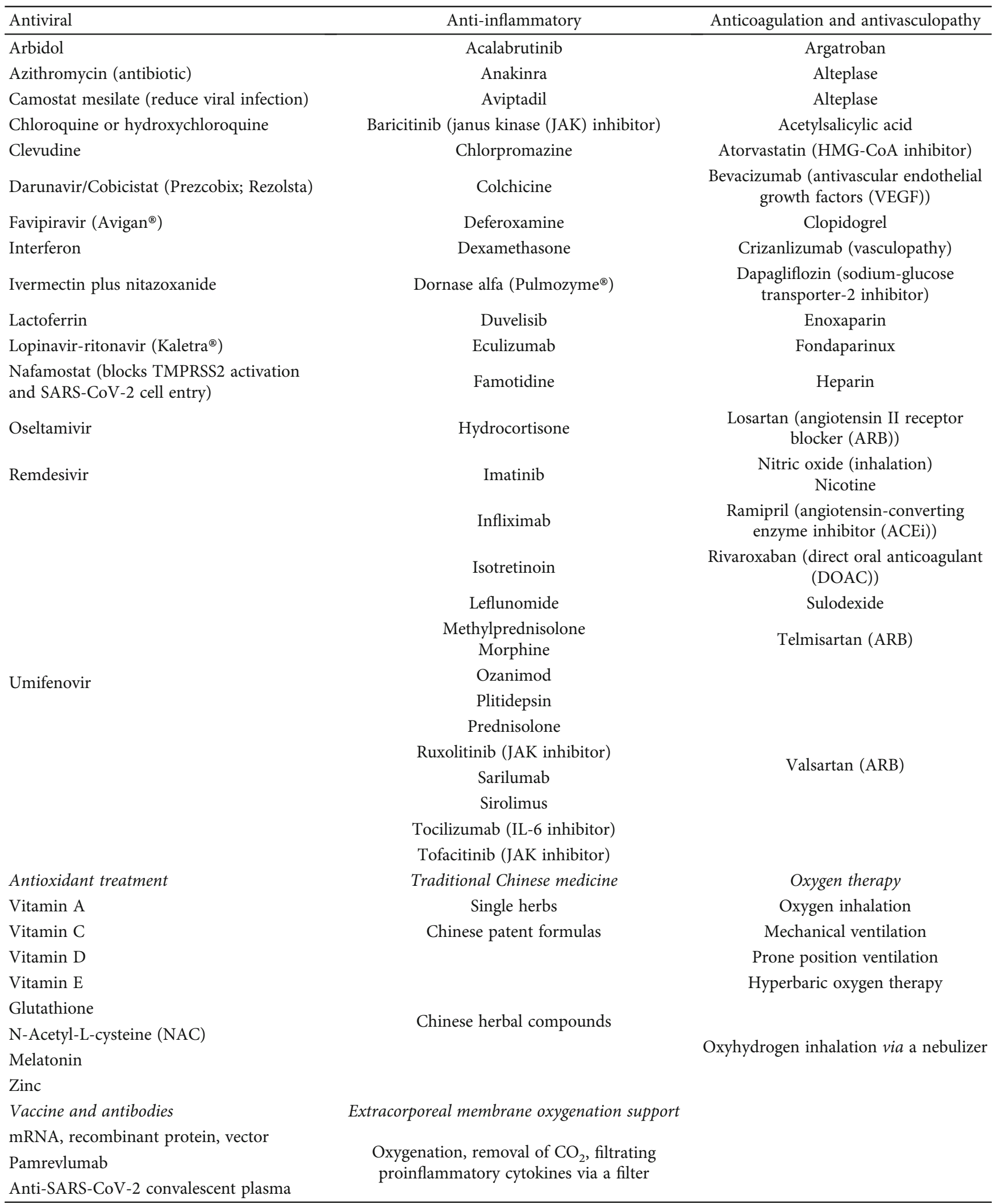

${ }^{*}$ Selected from 9149 total COVID-19 studies, including 6115 from COVID-19 NIH registered clinical trials [5] and the rest registered outside of the USA found from WHO International Clinical Trials Registry Platform (ICTRP) database [6-8]. 
easily penetrate mucus plugs; in fact, high airflow may instead further condense the plugs. It is also speculated that the positive pressure of ventilation may break the alreadyfragile alveolar sacs [9]. Moreover, ventilation of highly concentrated oxygen in patients with low $\mathrm{SpO}_{2}$ levels may produce harmful superoxide free radicals like what happens in ischemia reperfusion.

\section{Treatments Proposed and Investigated for COVID-19}

Current guidelines for COVID-19 critical care involve general supportive measures such as hemodynamic support with a vasopressor (usually norepinephrine), corticosteroids to treat refractory shock, continuous renal replacement therapy (CRRT) or intermittent renal replacement (IRR) for acute renal failure, and mechanical ventilation to treat severe ARDS. However, the clinical benefit for patients with severe disease that requires aggressive oxygen management, such as invasive or noninvasive mechanical ventilation, high-flow oxygen, or ECMO, is uncertain. Given the high cost, procurement hurdles, and pending research, health agencies have restricted distribution to hospital systems for patients 12 years of age or older requiring supplemental oxygen without aggressive oxygen management [10].

No single pharmacotherapy has shown sufficient clinical efficacy for routine use; at the clinician's discretion, however, select patients with severe disease may receive a trial of remdesivir and/or immunomodulatory therapy (such as corticosteroids) [10].

2.1. Antiviral Therapies. Some preliminary studies suggest that antiretroviral remdesivir (Veklury ${ }^{\mathrm{TM}}$ ) may modestly shorten recovery time. However, despite its in vitro activity against SARS-CoV-2, its effect on mortality rate for patients with severe COVID-19 is uncertain [11-13]. Remdesivir, an adenosine analog, purportedly targets viral RNA to cause premature termination of reverse transcription [13] (Figure 1).

Other antivirals, such as lopinavir/ritonavir (Kaletra ${ }^{\circledR}$ ), oseltamivir, or ribavirin, showed no clinical benefit in mortality [14-16]. Some studies combining lopinavir/ritonavir and ribavirin, however, have suggested a reduction in mortality and ARDS risk [14, 15]. Anti-infectives chloroquine and hydroxychloroquine have been studied exhaustively with clinical evidence suggesting no mortality benefit yet potential harm due to cardiac conduction abnormalities [17]. These results were negative despite their potent in vitro inhibitory effect on SARS-CoV-2 by raising host endosomal $\mathrm{pH}$ and preventing viral entry [13], though a study exploring their prophylactic role in healthcare workers is currently ongoing (NCT04334148). With similar publicity, the role of azithromycin remains contentious with the COALITION II trial, suggesting no clinical benefit when combined with hydroxychloroquine [18].

2.2. Immunomodulatory Therapies. Given the lack of effective antiviral treatments, some groups have investigated convalescent plasma $(\mathrm{CP})$ as an interim treatment. Historically,
$\mathrm{CP}$ has been used for various other infections (such as diphtheria, hepatitis $\mathrm{A}$ and $\mathrm{B}$, rabies, or polio) for which at some time periods, like COVID-19, lacked any suitable pharmacological treatment [19]. In theory, immunocompetent COVID-19 survivors could produce immunoglobins as part of acquired immunity, which can then be purified and transfused. Its efficacy is heralded by reports that reinfection with COVID-19 is rare, indicating that these antibodies may be highly effective in preventing or treating severe COVID-19 [20]. While some preliminary studies have demonstrated reduced mortality, reduced oxygen requirements, and reduced viral load, with mostly minor adverse events, large-scale and high-quality clinical research is lacking [20]. Furthermore, some hypothesize that, as with infections similar to SARS and Middle East respiratory syndrome (MERS) [21, 22], conferred immunity will only last for a limited number of months and may not be effective in the long term. With the lack of viral-targeted treatments, the clinical focus has since shifted more towards preventing complications in advanced disease, with promise in treating with corticosteroids.

Corticosteroids were previously avoided due to the potential decrease in immune responses and viral clearance and increase in osteopenia and osteoporosis observed in patients with SARS and MERS [23]. Preliminary studies, however, have suggested that corticosteroids may mitigate the sequelae that lead to multisystem organ failure and lung injury observed in severe COVID-19. In particular, clinicians have closely observed the preliminary results of an open-label trial, RECOVERY $(n=4321)$, which suggested a clinically significant decrease in mortality for patients requiring oxygen and ventilation when treated with a 10 day course of dexamethasone $6 \mathrm{mg}(\mathrm{NNT}=8$ for ventilated patients, 34 for nonventilated oxygen therapy). No mortality benefit was observed for patients with early disease, or mild to moderate disease not requiring oxygen therapy, suggesting that dexamethasone works against the inflammatory response in later stages of disease rather than reducing the viral load [24]. Other corticosteroids were also briefly studied and are used clinically with benefit [25], but were stopped early pending the RECOVERY trial publication: these included hydrocortisone in the REMAP-CAP and CAPE COVID trials [26] and methylprednisolone [27]. Given the promiscuous anti-inflammatory nature and risks of corticosteroids, including dysglycemia, immunosuppression, latent infection reactivation particularly with Strongyloides [28], and agitation, research interest blossomed in pharmacotherapies that target specific anti-inflammatory pathways.

Clinicians have reported cytokine storms manifesting in patients with severe COVID-19, which has promoted additional research into molecules that target proinflammatory pathways to treat ARDS and multiorgan sequelae [29]. Some of these molecules include interleukins, such as anakinra (anti-IL-1), aviptadil (anti-IL-6 and antitumor necrosis factor (TNF)), monoclonal antibodies (anti-IL-6; tocilizumab, sarilumab, and siltuximab), and JAK inhibitors (anti-IL-6; ruxolitinib baricitinib); general anti-inflammatories such as colchicine; and steroid-sparing immunosuppressives such 
as sirolimus and tacrolimus. Some of these studies have suggested potential clinical benefit in COVID-19. For instance, anakinra $5 \mathrm{mg} / \mathrm{kg}$ twice daily may improve survival for patients with moderate to severe ARDS compared to a historical cohort [30]. Similarly, studies with tocilizumab for patients experiencing cytokine storms have suggested potential benefit with one or two doses of 400 to $800 \mathrm{mg}$ [31, 32]. However, treatment with tocilizumab in some cases worsened COVID-19 infections, likely because of immunosuppression [33]. Similarly, studies with other molecules have suggested no clinical effect or potential harm due to immunosuppression (such as with sarilumab) [34], or insufficient power of statistical analysis to measure a mortality benefit (such as with colchicine) [35].

\subsection{Therapies with Ancillary Benefits from Other} Mechanisms of Action. Molecules targeting other host pathways are also being investigated, and many studies, as shown in Table 1, are still ongoing. Murine studies have suggested, for instance, that lung sequelae such as leukocyte infiltration and acute lung failure from the related SARS-CoV from the 2003 pandemic could be reduced with angiotensin II receptor blocker (ARB) losartan $15 \mathrm{mg} / \mathrm{kg}$, secondarily to inhibiting ACE2 binding of viral Spike-Fc [36]. Similarly, famotidine, a histamine 2-receptor blocker used for treating acid reflux disease, may inhibit viral replication by a mechanism still being investigated. Famotidine therapy was correlated with reduced inpatient mortality or intubation [37], with some cases of reduced outpatient symptom severity reported [38]. Furthermore, recent developments in coagulopathy secondarily to cytokine storms that expose the basement membrane and activate coagulation cascades have honed research in targeting VEGF (with bevacizumab), tissue plasminogen activators (alteplase) [39], and anticoagulants (argatroban, enoxaparin, fondaparinux, heparin, and rivaroxaban) [40].

Interestingly, some molecules have been investigated based on retrospective observations of patients with polypharmacy. Many of which seem to correlate with drugs that reduce inflammation and oxidative stress. For instance, some studies have suggested that sodium-glucose cotransporter-2 (SGLT2) inhibitors, a class of multifunctional antihyperglycemics, may prevent respiratory failure associated with endothelial disruption, inflammation, and oxidative stress by purportedly reducing serum lactate production and cytokines. Studies with dapagliflozin in patients with or without diabetes are currently underway (NCT04350593) [41]. Additionally, past studies with antilipidemic "statin" drugs (e.g., atorvastatin) have suggested improved symptom management in patients with concurrent viral infections with the annual avian influenza and the $2009 \mathrm{H} 1 \mathrm{~N} 1$. These effects may be ascribed to their antiinflammatory, antioxidant, and ACE2-downregulatory effects, which have prompted further clinical studies with atorvastatin [42]. In fact, this projection may be supported by observations from the use of statins in, for instance, the prevention of cytokine and oxidative stress-mediated iodinated contrast-induced nephrotoxicity [43].

Some think tanks have considered incidental findings of morphine and its inhibitory effects on cytokine production, particularly in dyspneic patients. Studies have found decreased levels of IL-12, TNF, and interferons, albeit inconsistently, when morphine is used in patients with chronic obstructive pulmonary disease (COPD) [44]. Other effects observed from morphine use may be translatable to similar features in the collection of syndromes related to COVID-19. One such study explored the prevention of mitochondrial-related reperfusion injury secondarily to postmyocardial infarction percutaneous intervention. By preventing the influx of reactive oxygen species and eventual cell death, morphine could have some effect in preventing damage after restoration of oxygen status to cells [45]. Studies with the use of morphine in dyspnea have been recruiting at the time of this article (NCT04522037).

Despite the current developments outlined, and over 9100 registered clinical trials to date [5-7], the vast research vision has tunneled to individual mechanisms that include viral entry, replication inhibition, or cytokine attenuation [46].

\section{Importance and Possible Mechanisms of Molecular Hydrogen in COVID- 19 Treatment}

Alveolar hypoxia, alveolar macrophages, and reactive oxygen species (ROS) cause an inflammatory response which may lead to ARDS. Excess proinflammatory cytokine secretion may further damage multiple organs. To address all of these contributing factors to cytokine storm in COVID-19, inhalation of molecular hydrogen may offer an effective solution to tackle both hypoxia and oxidative stress, thereby reducing downstream cytokine secretion. Many reports described possible mechanisms of molecular hydrogen actions against different diseases [47-60]. The majority of these reports revealed three main effects of molecular hydrogen in pathophysiology: antioxidative stress, antiinflammatory, and antiapoptotic effects. However, these three categories also include many subgroups of different effects of molecular hydrogen observed in various studies, for example, the regulation of oxidative stress, regulation of endoplasmic reticulum stress, regulation of mitochondria, inhibition of overactivation of the immune system, prevention of apoptosis, regulation of autophagy, reduction of pyroptosis-related inflammation, protection of cells from pyroptosis, positive regulation of ferroptosis, and potential regulation of the circadian clock. In 2020, Yang et al. listed the possible mechanisms of molecular hydrogen in 10 main disease systems [48]. In 2011, Ohta summarized the diseases and the organs targeted by molecular hydrogen treatment. After the appearance of the COVID-19 disease, many global efforts were applied to fight this pandemic [61]. In China, the famous epidemiologist Dr. Zhong Nanshan has applied $\mathrm{H}_{2} / \mathrm{O}_{2}$ inhalation for treating more than 2000 COVID-19 patients with very positive and effective outcomes [62, 63]. Additionally, a global scientific discussion has been launched on the ResearchGate platform about the possibility of the use of molecular hydrogen in COVID-19 treatment [64]. Several articles have been published about the potential benefits of molecular hydrogen therapy for COVID-19 [48, 65, 66], 
including its ability to combat effects of fatigue [67]. Although its beneficial effects have been reported in the literature and demonstrated in some clinical trials, a systemic review of the properties and underlying mechanisms of molecular hydrogen is necessary to broaden the utility of its positive effects in treating COVID-19. Currently, there is no report that fully elucidates the mechanisms behind the positive influence of molecular hydrogen in COVID-19 treatment.

\section{Physical, Chemical, and Biological Properties and Safety of Molecular Hydrogen}

4.1. Physical Properties of Molecular Hydrogen. Hydrogen is the most abundant element in the universe especially in stars. It combines with another hydrogen atom to form molecular hydrogen, with the chemical symbol of $\mathrm{H}_{2} . \mathrm{H}_{2}$ is the smallest and lightest molecule with a density of $0.08988 \mathrm{~g} / \mathrm{L}$ at standard temperature and pressure (STP). However, molecular hydrogen is rare in Earth's atmosphere at a level of about $0.53 \mathrm{ppm}$ [68]. Hydrogen is physically characterized as a nontoxic, colorless, odorless, tasteless, and nonmetallic gas at standard temperature and pressure. $\mathrm{H}_{2}$ has a lower solubility in water compared to oxygen and carbon dioxide with $0.8,1.3$, and $34.0 \mathrm{mmol} / \mathrm{L}$ at $20^{\circ} \mathrm{C}$, respectively [69]. The hydrogen-saturated water contains $0.78 \mathrm{mM}(1.6 \mathrm{mg} / \mathrm{L})$ of hydrogen at $25^{\circ} \mathrm{C}$. It was estimated that $2-5 \%$ of $\mathrm{H}_{2}$ is lost every $3 \mathrm{~min}$ when hydrogen-rich water is kept in an open container [70]. To preserve the levels of hydrogen in hydrogen-rich water during storage, the product must be filled in a metal package such as aluminum as plastics are permeable to $\mathrm{H}_{2}$ [51].

4.2. Chemical Properties of Molecular Hydrogen. The earliest known chemical property of hydrogen is that it burns with oxygen to form water. Under ordinary conditions, hydrogen gas is a loose aggregation of hydrogen molecules, each molecule consisting of a pair of hydrogen atoms, to form the diatomic molecule, $\mathrm{H}_{2}$ [71]. Additionally, molecular hydrogen can react with many elements and compounds, but at room temperature, the reaction rates are usually so low as to be negligible due to its very high dissociation energy [72].

In food processing, $\mathrm{H}_{2}$ is classified as a food additive with E949, and in the European Union (EU), it is permitted in part C group I of regulation 1129/2011 additives permitted at quantum satis [73]. At normal temperature and pressure, $\mathrm{H}_{2}$ is considered a noncorrosive and not very reactive substance (inert gas). It is used to store foodstuffs in packages under modified atmosphere beside $\mathrm{CO}_{2}$ and $\mathrm{N}_{2}$, and so protects them from undesirable chemical reactions such as food spoilage and oxidation during subsequent transport and storage [74, 75]. The addition of molecular hydrogen, i.e., hydrogenation, is used to produce margarine and vegetable shortening by converting unsaturated liquid animal and vegetable oils and fats to a saturated solid form. These processes require a catalyst, and high temperatures and pressures to overcome the activation energy of the stable nonpolar covalent bond that holds the hydrogen atoms together. Moreover, hydrogen is used to reduce aldehydes, fatty acids, and esters to the corresponding alcohols.
4.3. Biological Properties of Molecular Hydrogen. Intestinal bacteria in humans naturally produce hydrogen at about 50 to $1,000 \mathrm{mg} /$ day $[76,77]$ via degradation of oligosaccharides [78]. However, the amount of $\mathrm{H}_{2}$ produced by colonic fermentation is partially consumed by bacterial flora in the colon [70]. The ingestion of hydrogen-rich water was reported to increase both hydrogen peaks and the area under the curve (AUC) of breath hydrogen in a dose-dependent manner [79] within $10 \mathrm{~min}$ of ingestion [70]. It was estimated that approximately $41 \%$ of ingested $\mathrm{H}_{2}$ via hydrogen-rich water was kept in the body [70]. The loss of $\mathrm{H}_{2}$ from the skin surface is negligible (less than $0.1 \%$ ). Hydrogen may be transferred to the milk when the mother drinks hydrogen-rich water [80]. $\mathrm{H}_{2}$ has no adverse effects on the saturation level of arterial oxygen $\left(\mathrm{SpO}_{2}\right)$ and hemodynamic parameters [81]. The inhalation of $\mathrm{H}_{2} / \mathrm{O}_{2}$ mixed gas did not interfere with any vital signs of the body including respiratory rate, heart rate, blood pressure, and pulse oximetry [82].

4.4. Safety Property of Molecular Hydrogen. The American Conference of Governmental Industrial Hygienists classifies hydrogen as a simple asphyxiant and describes its major hazard due to its flammable and explosive properties [83]. Hydrogen is highly flammable at a range of $4-75 \%(v / v)$ in air, and it explodes in the air at the range of $18.3-59 \%(v / v$ ) $[84,85]$. However, the dilution of hydrogen with nitrogen lowers the risk of explosion [86]. Additionally, the autoignition temperature (the temperature at which spontaneous combustion will occur) of hydrogen is quite high, i.e., $500^{\circ} \mathrm{C}$.

\section{Redox-Related Mechanisms in the Pathophysiology of COVID-19}

The cellular redox status can affect the structural composition of various sensitive components found inside or on the surface of the cell. These redox-sensitive components include many proteins/enzymes composed of sulfurcontaining amino acids/peptides (SH and S-S) making them sensitive to the redox state of the environment. Methionine, cysteine (Cys), cystine, homocysteine, glutathione, and hydrogen sulfide are the common sulfur-containing compounds impacting protein regulation and cell signaling. Furthermore, the cofactors such as $\mathrm{Fe}, \mathrm{Zn}, \mathrm{Mg}$, and $\mathrm{Cu}$ found in their oxidized or reduced form, make the cellular enzymes susceptible to the redox change in the environment. In the same manner, we can discuss the effect of redox value on various redox-sensitive molecules located on the surface of the cell such as enzymes, proteins, phospholipids, and saturated and unsaturated fatty acids, which could become targets for the redox change in the environment/cytoplasm. The modification in the structure of these components can directly affect different functional and structural cellular systems such as cellular transport and bioenergetics.

The cell possesses a redox homeostasis system that regulates many key functions such as protein synthesis, enzyme activity, metabolic pathways, and transport across the membrane. This redox homeostasis can be regulated by different factors such as oxidoreductases (catalase (CAT), superoxide 
dismutase (SODs), and glutathione peroxidase (GPXs)), metallic ions ( $\mathrm{Fe}, \mathrm{Cu}, \mathrm{Mg}$, etc.), metabolites (adenosine triphosphate/adenosine monophosphate (ATP/AMP), glyceraldehyde 3-phosphate dehydrogenase (GAPDH), and tricarboxylic acid cycle (TCA) intermediates), gaseoussignaling molecules (ROS, $\mathrm{H}_{2}, \mathrm{H}_{2} \mathrm{~S}, \mathrm{CO}, \mathrm{NO}^{\circ}$, etc.), and internal antioxidants (ascorbate, vitamin $\mathrm{E}, \beta$-carotene, urate, and thiols). Amino acids and their macromolecules, i.e., peptides and proteins, can affect and be affected by the redox state of the cytoplasm and environment. The amino acids, peptides, and proteins containing thiols $(\mathrm{SH})$ form the targets for oxidants such as ROS [49]. The production of ROS and/or the change in the thiols/disulfide ratio lead to the perturbation of the intracellular redox homeostasis. This critical situation leads the cell to sense redox signaling, and thus regulate the cellular redox state [49]. When the levels of the generated ROS are high, the cell can use the redox-sensitive signaling pathways and transcription factors to upregulate genes encoding reductants such as thiols, enzymes, thioredoxin (Trxs), and glutaredoxins (Glrxs) that will reset redox homeostasis [49]. However, when the situation is more severe with very high levels of ROS, for example, during acute injury or inflammation, damage occurs to various macromolecules and cellular structures and functions, which can lead to irreversible injury and cell death. The presence of molecular hydrogen in the last case can mitigate the cytotoxic effects of ROS by reducing only the most aggressive ones, i.e., ${ }^{\circ} \mathrm{OH}$ and $\mathrm{ONOO}^{-}$, without affecting the physiologically beneficial ROS-dependent signaling molecules, i.e., $\mathrm{O}_{2}^{\cdot-}, \mathrm{H}_{2} \mathrm{O}_{2}$, and ${ }^{\circ} \mathrm{NO}$, and thus, maintaining redox homeostasis of the cell [52].

The modification of the structural composition of proteins due to the change of thiol (SH) to the disulfide (S-S) form impairs molecular chaperoning, translation, metabolism, cytoskeletal structure, cell growth, and signal transduction. Additionally, the formation of disulfide bonds affects the conformation of redox-sensitive proteins [58]. It was reported that in an oxidizing medium, the sulfur group in cysteine can form intramolecular disulfide bonds creating a reversible cross-link that can be broken in the presence of a reducing agent [87]. Oxidative stress conditions are characterized by a high generation of ROS and are related to many diseases involving disulfide bond formation [87]. Thiol-disulfide reactions follow an exchangeable and ratedependent bond rupture mechanism [87].

5.1. Importance of Thiols for Cellular Redox Status. Thiols have been shown to play a key role in many functional processes in cellular physiology. Glutathione (GSH), for example, was identified as a crucial intracellular antioxidant thiol that plays an essential role in protection against environmental oxidant-mediated injury in addition to its role in the redox signaling process [88]. The increase in the intracellular content of GSH leads to a decrease in the release of cytokines and chemokines from lung cells by decreasing $\mathrm{NF}-\kappa \mathrm{B}$ activation. This property was related to the antioxidant activity of GSH [88]. Normally, glutathione disulfide (GSSG) represents less than $1 \%$ of the total cellular GSH pool. The perturbation to the GSH/GSSG ratio due to the excessive generation of ROS can alter signaling pathways that play key roles in many physiological responses such as cell proliferation, autophagy, apoptosis, and gene expression.

It was reported that the activation of redox-sensitive transcription factors such as nuclear factor erythroid 2related factor 2 (Nrf2), NF- $\kappa \mathrm{B}$, and activator protein 1 (AP-1) differentially regulate the genes for proinflammatory cytokines as well as the protective antioxidant genes [88]. Moreover, GSH is considered a crucial factor for the enzymatic activity of GPx, which is a major contributor to the cellular enzymatic antioxidant defense [89]. Sustained oxidative challenge leads to depletion of lung GSH along with other antioxidants forming the main reasons for many lung diseases, e.g., ARDS, chronic obstructive pulmonary disease (COPD), asthma, cystic fibrosis (CF), idiopathic pulmonary fibrosis (IPF), and neonatal lung disease [88]. Moreover, GSH levels were found to be depleted in several viral infections such as infection with HIV, influenza A virus, hepatitis $\mathrm{C}$ virus, and herpes simplex virus-1 [90]. On the other hand, the decrease in the levels of GSH in the lung lining fluid have been shown in various pulmonary diseases such as IPF, ARDS, CF, lung allograft patients, and patients with human immunodeficiency virus (HIV) [88]. This observation was explained by the formation of disulfide bonds due to the huge generation of ROS. Accordingly, several approaches have been studied to increase the cellular GSH levels to improve the cell's ability to cope with the increased ROS production. The administration of GSH itself has been shown to have limited therapeutic value due to its short plasma half-life, i.e., $<30 \mathrm{~min}$, and its inability to pass the cell membrane. Therefore, other strategies have been evaluated to increase intracellular GSH pools.

One of the most studied pro-GSH molecules is N-acetylL-cysteine (NAC). Roederer et al. demonstrated in 1992 that NAC inhibited HIV replication in vitro [91]. NAC, ascorbic acid, and vitamin $\mathrm{E}$ were reported to decrease both viral replication and inflammation in cells of mice infected with influenza (IV) and/or human respiratory syncytial (HRSV) respiratory viruses [92]. Although the treatment of NAC in vitro and in vivo experiments showed an increase in GSH levels that reduced the viral load by inhibiting viral replication in several viruses, e.g., influenza A ( $\mathrm{H} 3 \mathrm{~N} 2$ and $\mathrm{H} 5 \mathrm{~N} 1$ ), the protective effect of NAC alone appeared weak or null in some models with a variation in its efficacy depending on the infecting viral strain [93]. Based on a trial study of 198 patients with COVID-19, a noticeable increase of glutathione reductase levels occurred in around $40 \%$ of COVID-19 patients [93] suggesting an increase in GSH metabolism. However, although NAC may be effective in this case, its antioxidant and therapeutic benefits may be strain specific. Therefore, clinical evidence is required before NAC supplementation can be recommended. Moreover, there is currently no COVID-specific evidence for the use of NAC [93].

5.2. Potential Use of Molecular Hydrogen to Improve Cellular Redox Status. A favorable GSH balance was reported to ameliorate bronchial asthma by suppressing chemokine production and eosinophil migration itself [88]. The latter authors 
TABLE 2: Summary of some possible mechanisms related to the positive effects of molecular hydrogen in different diseases and COVID-19 treatment.

\begin{tabular}{lcl}
\hline Possible Mechanism & $\begin{array}{c}\text { Type of } \\
\text { Study }\end{array}$ & Principle \\
\hline
\end{tabular}

Molecular properties-related mechanisms

Redox-related mechanisms

Inflammatory reactions and apoptosis-related mechanisms

Animal

In vivo

In vivo

in silico

Animal
Reduces the reversible cross-linked intramolecular disulfide bonds formed after cytosol, mitochondria and nucleus and reach cell organelles

Has a rapid gaseous diffusion rate making it highly effective for reducing cytotoxic radicals

Regulates the redox homeostasis after a ROS-related dissipation stage

Mild enough not to disrupt metabolic oxidoreduction reactions or interrupt ROS-induced disruption of cell signaling

Selectively reduce the strongest cytotoxic oxidants, ${ }^{\bullet} \mathrm{OH}$ and $\mathrm{ONOO}^{-}$; whereas, the biological useful oxidants such as superoxide, hydrogen peroxide, nitric oxide are not altered

Protects nuclear DNA and mitochondria

Protects cells and tissues against strong oxidative stress

Decreases production of ROS an oxidative stress e.g. ROS

Decreases the energy barrier of disulfide rupture

Balances the S-S/SH in favor of thiols

Protects Inositol 1, 4, 5-trisphosphate receptors (IP3Rs) function

Protects the ATP-induced $\mathrm{Ca}^{2+}$ signal by reducing the $\mathrm{H}_{2} \mathrm{O}_{2}$-induced disulfide bonds in IP3Rs and restores protein function

Activates glutathione/thioredoxin systems involved in the modulation

of disulfide bond formation during oxidative stress leading to reduced $\mathrm{H}_{2} \mathrm{O}_{2}$-induced disulfide bond formation

Repairs the processes of cell injury produced through high ROS generation

Mitigates the oxidative damage

selectively reduces ${ }^{\bullet} \mathrm{OH}$ attenuating ischemia/reperfusion-Induced organ damage

Animal Increases superoxide dismutase (SOD) activity against ROS-mediated cellular damage

Increases activities of antioxidant enzymes

Can significantly decrease levels of oxidative products

Human Induces superoxide dismutases (SODs) activity to quench ROS production

Decreases ROS levels via upregulating superoxide dismutase (SOD) and

Human glutathione (GSH) as well as downregulating NADPH oxidase (NOX 2) expression

Decreases oxidative damage

Inhibits the over-expression of inflammatory factors (IL-6, IL-8 and TNF- $\alpha$ )

Downregulates the expression of proapoptotic Fas proteins

Up-regulates the expression of the anti-apoptotic protein Bcl2

Ameliorates LPS-induced bronchopulmonary dysplasia

Reduces LPS-induced oxidative stress production

Ameliorates LPS-induced suppression of genes encoding fibroblast growth factor receptor 4 (FGFR4), VEGFR2, and HO-1, as well as LPS-induced overexpression of inflammatory marker proteins (TNF $\alpha$ and IL-6)

Lung and alveoli-related

Animal Suppresses the induced expressions of inflammatory marker proteins mechanisms

Reduces ROS production in alveolar epithelial cells

Animal 
TABLE 2: Continued.

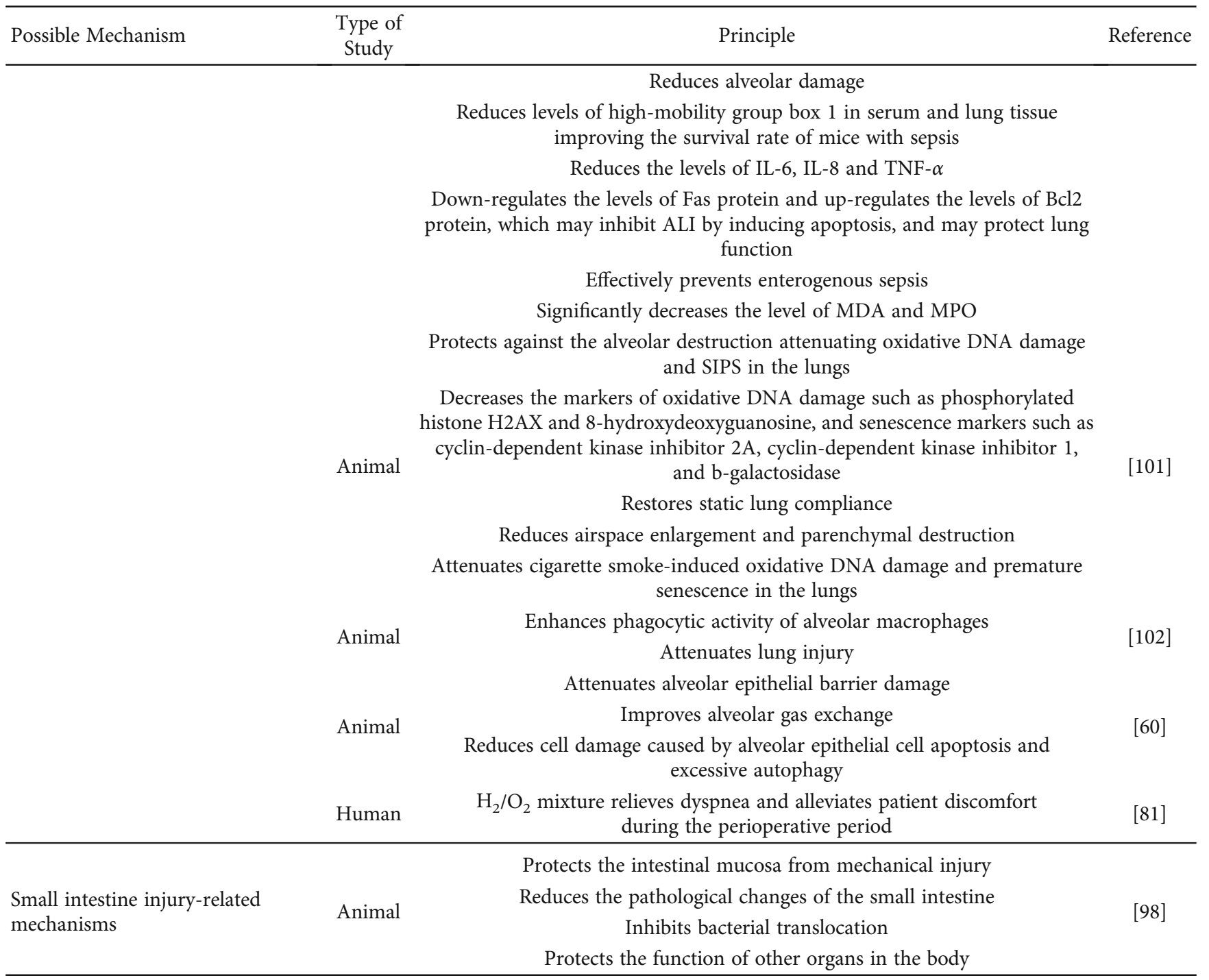

revealed that small changes in the cellular redox status may alter signaling pathways, and the GSH/GSSG ratio can serve as a good indicator of the cellular redox state. While the increase in the GSH/GSSG leads to proliferation, the decrease in the GSH/GSSG causes apoptosis. GSH/GSSG and Cys/CySS were found to be decreased in some oxidative-related diseases such as smoking, diabetes, obesity, and pneumonia [94]. Those most susceptible to developing COVID-19 and serious illness are those with underlying pathologies such as obesity, which is associated with impaired redox and inflammatory homeostasis [95]. Another beneficial role of hydrogen in oxidative stress-related diseases may be attributed to balancing the S-S/SH in favor of thiols. Previous reports indicate that the presence of reducing agents decreased the number of disulfide bonds, resulting in a loss of cross-link-induced stability produced by the chemical microenvironment [58]. In 2012, Keten et al. reported that the stability of the disulfide bond may mildly be influenced by the redox value of the chemical microenvironment where the concentration of reducing agents can trigger various fractures in the protein by decreas- ing the energy barrier of disulfide rupture [87]. They performed a simulation of disulfide rupture in the presence of a hydrogen molecule, illustrating the reduction mechanism of the disulfide bond. This phenomenon was explained by the elongation of the disulfide bond leading to a weakening of the bond followed by a reduction of the sulfur atoms and fracture of the protein at the S-S bond. The authors assumed that the reaction of the hydrogen molecule with a disulfide bond occurs violently once they are near each other [87] (Table 2). However, there is no evidence that this hypothetical mechanism is responsible for the observed biological effects of molecular hydrogen at improving the GSH/GSSG ratio. However, $\mathrm{H}_{2}$ can increase GSH levels [96] by activating the Nrf2 pathway [97]. A nonsignificant increase in GSH, GSH/GSSG, and GSH peroxidase combined with a decrease in GSSG levels in rat livers fed with hydrogen-rich water compared to control was reported [98].

Interestingly, both endogenous and exogenous oxidants have been shown to need hours to significantly affect GSH levels in the majority of cells [88]. This is a double-edged 


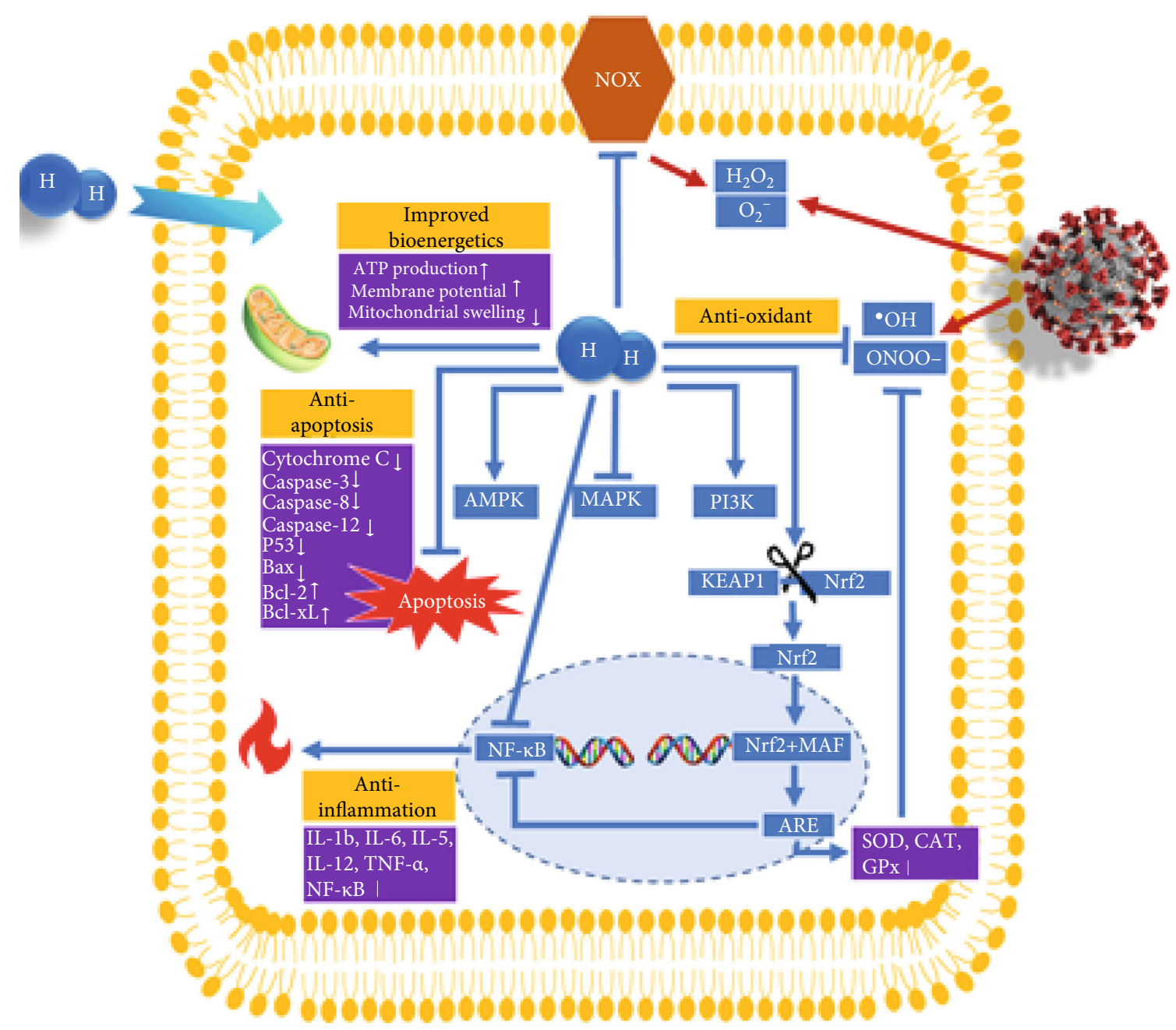

Figure 2: Possible mechanisms of alleviation properties of molecular hydrogen on COVID-19 patients. ${ }^{\bullet} \mathrm{OH}$ : hydroxyl radical; $\mathrm{O}_{2}^{-}$: superoxide anion; $\mathrm{ONOO}^{-}$: peroxynitrite anion; $\mathrm{H}_{2} \mathrm{O}_{2}$ : hydrogen peroxide; $\mathrm{H}_{2}$ : molecular hydrogen; NOX: nicotinamide adenine dinucleotide phosphate (NADPH) oxidase; AMPK: $5^{\prime}$ adenosine monophosphate- (AMP-) activated protein kinase; MAPK: mitogenactivated protein kinase; PI3K: phosphatidylinositol 3-kinase; Keap1: Kelch-like ECH-associated protein 1; Nrf2: nuclear factor erythroid 2-related factor 2; MAF: small MAF protein; ARE: Nrf2-antioxidant response element; SOD: superoxide dismutase; CAT: catalase; GPx: glutathione peroxidase; P53: tumor protein; Bax: BCL2-associated X protein; Bcl-2: B-cell lymphoma 2 protein; Bcl-XL: B-cell lymphoma-extra-large protein; IL-12: interleukin 12; IL-1 $\beta$ : interleukin 1-beta; IL-6: interleukin 6; IL-8: interleukin 8; TNF- $\alpha$ : tumor necrosis factor $\alpha$; NF- $\kappa$ B: nuclear factor-kappa-light-chain-enhancer of activated B cells.

sword because, on the one hand, the redox status stays in the range of homeostasis despite a significant amount of oxidative stress. On the other hand, by the time the GSH/GSSG ratio has changed enough to be detected, it may be too late and/or too difficult to reestablish homeostasis by pharmacological interventions. Once the GSH levels are depleted, the antioxidant redox cycling is also negatively impacted, potentially rendering pharmacological interventions or antioxidant supplementation less effective. However, premature ingestion of reducing substances either orally or intravenously may exacerbate the redox condition. In contrast, molecular hydrogen is capable of reaching any organelle in the cell within minutes and does not perturb the GSH/GSSH ratio from optimal homeostasis. Instead, $\mathrm{H}_{2}$ modulates signal transduction and maintains optimal redox homeostasis within the cell (Table 2). In this way, $\mathrm{H}_{2}$ has the ability to act as a reducing agent at low concentration with the ability to antagonize the ROS-induced deleterious effects on cell signaling [50]. $\mathrm{H}_{2}$ has been characterized by its ability to decrease ROS levels via upregulating superoxide dismutase (SOD) and glutathione (GSH) as well as downregulating NADPH oxidase (NOX 2) expression in a rat model [101] (Table 2 and Figure 2).

An additional but crucial role of hydrogen was found in repair processes of cell injury produced through high ROS generation. $\mathrm{H}_{2}$ can induce heat shock proteins (HSPs) and suppress ROS production [58]. For example, the activation of glutathione/thioredoxin systems, which reduces $\mathrm{H}_{2} \mathrm{O}_{2}$ induced disulfide bond formation, is another possible mechanism underlying the $\mathrm{H}_{2}$-induced elimination of ROS damage of inositol 1,4,5-trisphosphate receptors (IP3Rs) [58]. $\mathrm{H}_{2} \mathrm{O}_{2}$ is a highly reactive molecule capable of oxidizing 
sulfhydryl groups of cysteine and methionine in proteins and forming sulfenic acid or disulfide $[49,58]$. This modification in the structure induces dysfunction of proteins leading to the impairment of many physiological processes. By this phenomenon, $\mathrm{H}_{2} \mathrm{O}_{2}$ was able to decrease the $\mathrm{Ca}^{2+}$ signal by triggering IP3R disulfide bond formation. However, the IP3R function was partially protected by treatment with $\mathrm{H}_{2}$ [58]. In other words, the $\mathrm{H}_{2}$-containing medium protected the ATP-induced $\mathrm{Ca}^{2+}$ signal by reducing the $\mathrm{H}_{2} \mathrm{O}_{2}$-induced disulfide bonds in IP3Rs.

SARS-CoV-2 infection was reported to evoke free radical-associated damage in the body by targeting different molecules. Therefore, all therapeutic means that can alleviate free radicals may be considered for COVID-19 patients to conquer the inflammation-induced burst of free radicals [104]. The rapid gaseous diffusion of $\mathrm{H}_{2}$ makes it highly effective for penetrating the subcellular compartments of the body. Importantly, $\mathrm{H}_{2}$ was identified as clinically more effective than two ROS scavengers for the treatment of cerebral infarction, i.e., edaravone and FK506, in alleviating oxidative injury [105]. In addition to the greater benefit compared to other ROS scavengers, $\mathrm{H}_{2}$ is considered mild enough not to affect the ROS that play essential roles in signal transduction such as $\mathrm{H}_{2} \mathrm{O}_{2}, \mathrm{NO}^{\bullet}$, and $\mathrm{O}_{2}^{-\bullet}$ [50]. $\mathrm{H}_{2}$ can react with only the strongest oxidants, i.e., ${ }^{\circ} \mathrm{OH}$ and $\mathrm{ONOO}^{-}$, which are considered the most reactive ROS (Figure 2). Additionally, $\mathrm{H}_{2}$ does not reduce the oxidized form of some biomolecules/cofactors involved in metabolic oxidoreduction reactions, e.g., $\mathrm{NAD}^{+}, \mathrm{FAD}$, or the oxidized form of cytochrome C [50] (Table 2).

\subsection{Alveolus-Related Mechanism of Molecular Hydrogen-} Based COVID-19 Treatment. Pulmonary surfactants play various crucial roles in the function of alveoli. The surfactants prevent lung collapse, increase the gas exchange, and contribute to the elastic properties of the lungs. These functions of surfactants can be accomplished due to their ability to reduce the surface tension inside the alveoli. These surfactants are composed of lipids, phospholipids, and proteins synthesized and secreted by alveolar type II cells that line the alveolar surfaces of the lungs [106]. The fluid lining alveolar surfaces contains different antioxidants such as GSH, vitamin C, and ceruloplasmin, which can quench free radicals [106]. The content of GSH in the respiratory tract lining fluids (RTLFs) was reported to be subnormal in various diseases such as acute immunodeficiency syndrome (AIDS), idiopathic pulmonary fibrosis, cystic fibrosis, acute respiratory disease syndrome, and in lung allograft patients [107]. The SOD and CAT were reported to be found in both surfactant and lung epithelial lining fluid, and take part in the regulation of postnatal lung vascular development and the protection of microvasculature from ROSinduced injury [108].

The oxidative modification of surfactants due to the effect of ROS on phospholipids, lipids, proteins, and biophysical activity can lead to dysfunction and several lung diseases such as acute lung injury and acute respiratory distress syndrome [109]. ROS production can lead to an increased lipid peroxidation and destruction of the cell membrane of the alveolar epithelial cells, and an increased membrane permeability [99].

Two factors were reported to promote the oxidation of surfactant lipids. First, the excessive production of ROS makes the antioxidant defenses incapable of providing protection. Secondly, the major antioxidants in the alveoli may be excluded from the microenvironment [106]. The ROS or reactive nitrogen species (RNS), especially $\mathrm{ONOO}^{-}$, produced during lung injury can cause surfactant inactivation leading to increased leakage of proteins into the alveoli [110]. This latter situation prolongs the need for supplemental oxygen and assisted ventilation. It was reported that, once the SARS-CoV-2 enters the respiratory tract, it reaches the alveoli where its primary target is the type II pneumocyte, thus impairing surfactant production [111]. It was reported that both SARS-CoV-2 and SARS-CoV-1 viruses perturb alveoli to produce the major pathology in the lung, resulting in increased fluid entry, cell death, and inflammation, along with a reduction in gas exchange and levels of surfactant [112] (Figure 3).

Different antioxidants were proposed to prevent lipid peroxidation of lung surfactants such as melatonin-ebselen and vitamin E [106]. Importantly, it was reported that the continuous exposure ( 24 hours) to $10 \%$ hydrogen decreased the production of ROS in A549 human lung epithelial cells [80]. It was also revealed that inhalation of $2 \%$ hydrogen attenuated septic shock-induced organ injury and decreased neutrophil infiltrate in the alveoli, and reduced alveolar damage [99]. On the other hand, inhalation of $\mathrm{H}_{2} / \mathrm{O}_{2}$ mixed gas has been shown to reduce the inspiratory effort in patients with acute severe tracheal stenosis [82]. Moreover, hydrogen-rich water was reported to protect against the alveolar destruction attenuating the oxidative DNA damage and swimming-induced pulmonary edema (SIPS) in the lungs of COPD model mice [102]. Furthermore, hydrogenrich water was found to attenuate lung injury by inhibiting lipid peroxidation [103]. Hydrogen-rich saline was also reported to reduce ROS production in alveolar epithelial cells, attenuate the alveolar epithelial barrier damage, improve alveolar gas exchange, and reduce cell damage caused by alveolar epithelial cell apoptosis and excessive autophagy [60] (Table 2).

\section{Conclusion and Perspectives}

An explanation for the advantageous effects of molecular hydrogen in COVID-19 treatment is related to the different properties of molecular hydrogen: (1) the small molecular size and nonpolarity of $\mathrm{H}_{2}$ allow it to rapidly permeate the tissues and cells, (2) it can selectively reduce only the cytotoxic ROS, (3) it can suppress the excessive production of otherwise good ROS, (4) it can suppress proinflammatory cytokines, (5) it can induce cytoprotective heat shock proteins, (6) it can improve mitochondrial bioenergetics, and (7) it has no known toxic effects even at very high levels [114]. These properties may explain the improvement in the conditions of COVID-19 patients treated by inhalation of $\mathrm{H}_{2} / \mathrm{O}_{2}$ mixed gas $\left(67 \% \mathrm{H}_{2} / 33 \% \mathrm{O}_{2}\right)$, who felt reduction in chest pain and cough, and easier deeper breathing and 


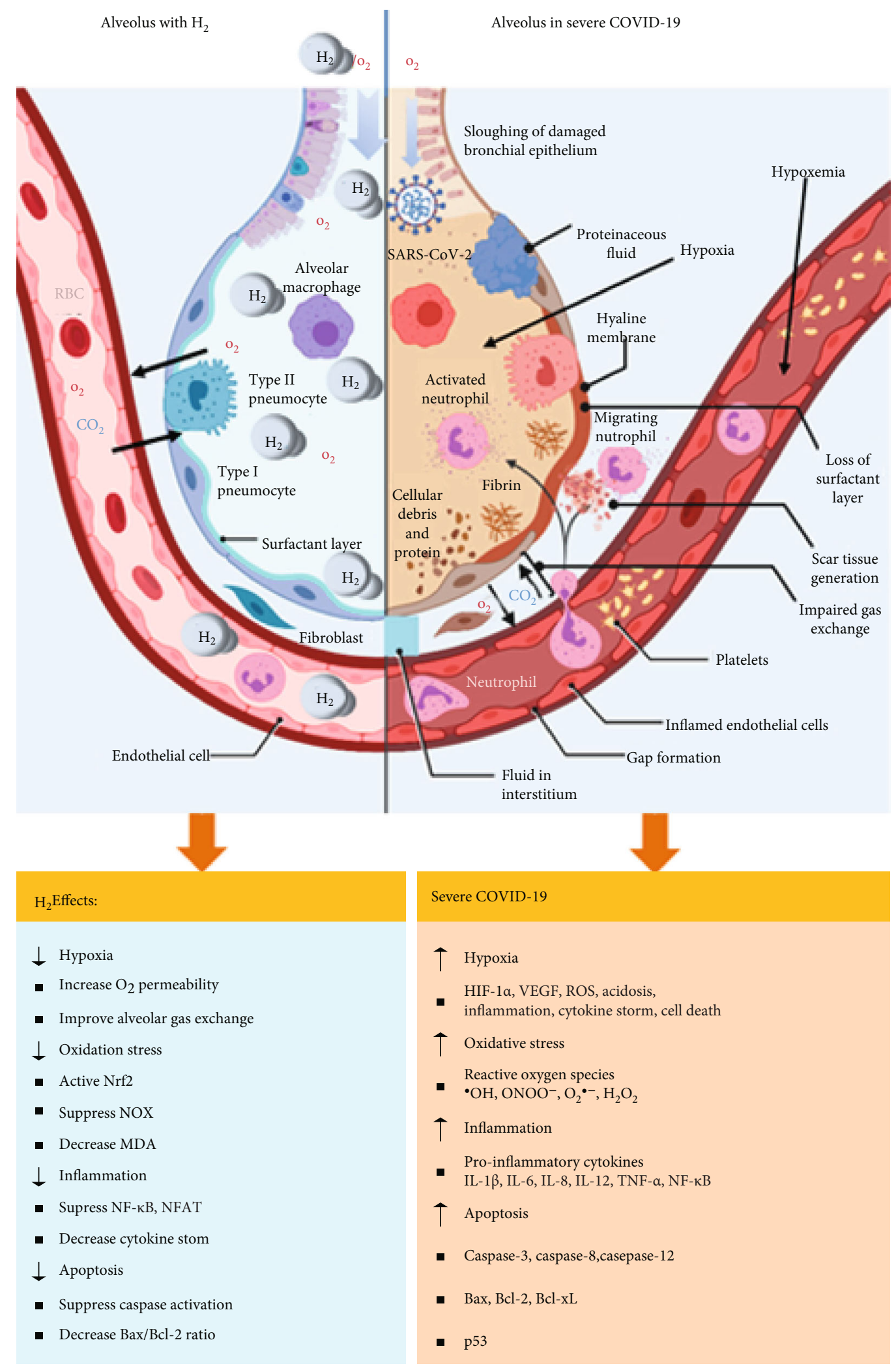

FIGURE 3: Alveolar changes due to SARS-CoV-2 infection in severe COVID-19 cytokine syndrome-induced acute respiratory distress syndrome (ARDS). Reproduced and modified from [113].

comfort sensation $[62,63]$. The positive results of the pilot study led Dr. Zhong Nanshan, the epidemiologist who discovered the SARS virus (SARS-CoV-1) in 2003, to recom- mend the $\mathrm{H}_{2} / \mathrm{O}_{2}$ inhalation therapy for COVID-19 patients [115] and prompted more clinical trials using $\mathrm{H}_{2} / \mathrm{O}_{2}$ mixed gas [116-118]. 
Currently, there are twenty registered clinical trials on the use of $\mathrm{H}_{2}$ for COVID-19. Of these, four are registered at the Centre for Evidence-Based Medicine (Oxford) using $\mathrm{H}_{2} / \mathrm{O}_{2}$ mixed gas inhalation [116], five clinical trials are registered at ClinicalTrials.gov of the US National Library of Medicine for inhalation [118], eight clinical trials are registered at ICTRP (WHO) with six for inhalation and two trials for hydrogen-rich water [8], and three clinical trials, related to the use of either inhalation or ingestion of hydrogen-rich water, are registered at the Chinese Clinical Trial Registry center [117]. Up to date, the reported benefits of $\mathrm{H}_{2}$ therapy in COVID-19 patients are limited to the symptomatic description. To expand the utility of $\mathrm{H}_{2}$ therapy in COVID-19, more thorough understanding of the underlying mechanism of $\mathrm{H}_{2}$ in patients is required. Therefore, accurate analysis of a broad spectrum of biomarkers is highly recommended to delineate the correlation between clinical and biochemical presentations and the proposed biological effect of $\mathrm{H}_{2}$.

According to the report of WHO, data from China and around the world suggest that the majority of people with COVID-19 have a mild illness, about $15 \%$ of them have a severe illness requiring oxygen therapy, and 5\% are critically ill requiring mechanical ventilation. Owing to the widespread transmissibility and emergence of more infectious variants of SARS-CoV-2, many hospitals have been overwhelmed by the crush of new COVID-19 patients and have exhausted ICU beds and ventilators in some regions. Therefore, an alternative yet effective treatment, e.g., $\mathrm{H}_{2} / \mathrm{O}_{2}$ gas inhalation, would ease the pressure on hospitals and prevent severe illness of COVID-19 patients.

The medical model of $\mathrm{H}_{2} / \mathrm{O}_{2}$ mixed gas machine is small, portable, and safe [119]. It costs about one-tenth of the price of a ventilator. The $\mathrm{H}_{2} / \mathrm{O}_{2}$ inhalation treatment may be performed in regular wards or by outpatients at home isolation using a portable $\mathrm{H}_{2} / \mathrm{O}_{2}$ generating and inhalation device. This kind of treatment may reduce hospitalization time for a high number of patients. This strategy could decrease the pressure of massive patient numbers on hospitals. It is important to mention that, although molecular hydrogen is not considered a drug, its intake in different ways such as drinking hydrogen-rich water or inhaling $\mathrm{H}_{2} / \mathrm{O}_{2}$ gas may be beneficial in preventive medical health in addition to its therapeutic use. Due to the high safety profile and favorable preliminary results in preclinical and clinical studies, application and additional research of molecular hydrogen therapy for COVID-19 are encouraged.

\section{Conflicts of Interest}

The authors declare that there is no conflict of interest regarding the publication of this paper.

\section{Acknowledgments}

The authors acknowledge Gerry Davies, Grace Russell, Geoff Taylor, Robert Bryant, Jack Silver, Danny Day, Vladimir L. Safonov, Christian S. Yorgure, Bird Kumar, Snehangshu Parta, George Wiseman, and other scientists and technolo- gists for actively participating in the ResearchGate Forum entitled "Potential Cheap and Effective Drug for COVID19 " and contributing their valuable comments. This work is partly supported by the Natural Sciences and Engineering Research Council of Canada (NSERC) and the Canada Council for the Arts Killam Research Fellowship to X.Y.W.

\section{References}

[1] WHO, Naming the Coronavirus Disease (COVID-19) and the Virus That Causes It, WHO, 2020.

[2] Johns Hopkins University, COVID-19 map, Johns Hopkins University, 2020.

[3] M. Cascella, M. Rajnik, A. Cuomo, S. C. Dulebohn, and R. Di Napoli, Features, evaluation and treatment coronavirus (COVID-19), StatPearls Publishing, 2020.

[4] J. Y. Chung, M. N. Thone, and Y. J. Kwon, "COVID-19 vaccines: the status and perspectives in delivery points of view," Advanced Drug Delivery Reviews, vol. 170, pp. 1-25, 2021.

[5] ClinicalTrials.gov Views of COVID-19 Studies Listed on ClinicalTrials.gov (Beta), U.S. National Library of Medicine, 2021.

[6] A. R. Bourgonje, A. E. Abdulle, W. Timens et al., "Angiotensin-converting enzyme 2 (ACE2),SARS-CoV-2 and the pathophysiology of coronavirus disease 2019 (COVID-19)," The Journal of Pathology, vol. 251, no. 3, pp. 228-248, 2020.

[7] C. Wang, J. Xie, L. Zhao et al., "Alveolar Macrophage Activation and Cytokine Storm in the Pathogenesis of Severe COVID-19," 2020.

[8] International Clinical Trials Registry Platform, "Important information about the COVID-19 outbreak," 2021, https:// www.who.int/clinical-trials-registry-platform.

[9] N. Ashraf-Kashani and R. Kumar, "High-flow nasal oxygen therapy," Bja Education, vol. 17, no. 2, pp. 63-67, 2017.

[10] Panel, C.-19 T. G, COVID-19 Treatment Guidelines Panel. Coronavirus Disease 2019 (COVID-19) Treatment Guidelines. National Institutes of Health, National Institutes of Health, 2020, https://www.covid19treatmentguidelines.nih.gov/.

[11] J. H. Beigel, K. M. Tomashek, L. E. Dodd et al., "Remdesivir for the treatment of COVID-19-final report," The New England Journal of Medicine, vol. 383, no. 19, pp. 18131826, 2020.

[12] J. D. Goldman, D. C. B. Lye, D. S. Hui et al., "Remdesivir for 5 or 10 days in patients with severe Covid-19," The New England Journal of Medicine, vol. 383, no. 19, pp. 18271837, 2020.

[13] M. Wang, R. Cao, L. Zhang et al., "Remdesivir and chloroquine effectively inhibit the recently emerged novel coronavirus (2019-nCoV) in vitro," Cell Research, vol. 30, no. 3, pp. 269-271, 2020.

[14] B. Cao, Y. Wang, D. Wen et al., "A trial of lopinavir-ritonavir in adults hospitalized with severe Covid-19," The New England Journal of Medicine, vol. 382, no. 19, pp. 1787$1799,2020$.

[15] Y. Song, M. Zhang, L. Yin et al., "COVID-19 treatment: close to a cure? A rapid review of pharmacotherapies for the novel coronavirus (SARS-CoV-2)," International journal of antimicrobial agents, vol. 56, no. 2, article 106080, 2020.

[16] D. Wang, B. Hu, C. Hu et al., "Clinical characteristics of 138 hospitalized patients with 2019 novel coronavirus-infected pneumonia in Wuhan, China," Jama, vol. 323, no. 11, pp. 1061-1069, 2020. 
[17] A. V. Hernandez, Y. M. Roman, V. Pasupuleti, J. J. Barboza, and C. M. White, "Hydroxychloroquine or chloroquine for treatment or prophylaxis of COVID-19," Annals of Internal Medicine, vol. 173, no. 4, pp. 287-296, 2020.

[18] R. H. M. Furtado, O. Berwanger, H. A. Fonseca et al., "Azithromycin in addition to standard of care versus standard of care alone in the treatment of patients admitted to the hospital with severe COVID-19 in Brazil (COALITION II): a randomised clinical trial," Lancet, vol. 396, no. 10256, pp. 959-967, 2020.

[19] M. M. Eibl, "History of immunoglobulin replacement," Immunology and Allergy Clinics of North America, vol. 28, no. 4, pp. 737-764, 2008.

[20] V. Piechotta, C. Iannizzi, K. L. Chai et al., "Convalescent plasma or hyperimmune immunoglobulin for people with COVID-19: a living systematic review," Cochrane Database of Systematic Reviews, no. 5, 2020.

[21] H. Mo, G. Zeng, X. Ren et al., "Longitudinal profile of antibodies against SARS-coronavirus in SARS patients and their clinical significance," Respirology, vol. 11, no. 1, pp. 49-53, 2006.

[22] D. C. Payne, I. Iblan, B. Rha et al., "Persistence of antibodies against Middle East respiratory syndrome coronavirus," Emerging Infectious Diseases, vol. 22, no. 10, pp. 1824-1826, 2016.

[23] C. D. Russell, J. E. Millar, and J. K. Baillie, "Clinical evidence does not support corticosteroid treatment for 2019-nCoV lung injury," Lancet, vol. 395, no. 10223, pp. 473-475, 2020.

[24] RECOVERY Collaborative Group, P. Horby, W. S. Lim et al., "Dexamethasone in hospitalized patients with Covid-19," The New England Journal of Medicine, vol. 384, no. 8, pp. 693-704, 2020.

[25] The WHO Rapid Evidence Appraisal for COVID-19 Therapies (REACT) Working Group, J. A. C. Sterne, S. Murthy et al., "Association between administration of systemic corticosteroids and mortality among critically ill patients with COVID-19: a meta-analysis," JAMA, vol. 324, no. 13, pp. 1330-1341, 2020.

[26] D. C. Angus, S. Berry, R. J. Lewis et al., “The REMAP-CAP (Randomized Embedded Multifactorial Adaptive Platform for Community-Acquired Pneumonia) Study. Rationale and Design," Annals of the American Thoracic Society, vol. 17, no. 7, pp. 879-891, 2020.

[27] Y. Wang, W. Jiang, Q. He et al., "A retrospective cohort study of methylprednisolone therapy in severe patients with COVID-19 pneumonia," Signal Transduction and Targeted Therapy, vol. 5, no. 1, p. 57, 2020.

[28] W. M. Stauffer, J. D. Alpern, and P. F. Walker, "COVID-19 and dexamethasone: a potential strategy to avoid steroidrelated Strongyloides hyperinfection," JAMA, vol. 324, no. 7, pp. 623-624, 2020.

[29] P. Mehta, D. McAuley, M. Brown et al., "COVID-19: consider cytokine storm syndromes and immunosuppression," Lancet (London, England), vol. 395, no. 10229, pp. 1033-1034, 2020.

[30] T. Huet, H. Beaussier, O. Voisin et al., "Anakinra for severe forms of COVID-19: a cohort study," The Lancet Rheumatology, vol. 2, no. 7, pp. e393-e400, 2020.

[31] N. Biran, A. Ip, J. Ahn et al., "Tocilizumab among patients with COVID-19 in the intensive care unit: a multicentre observational study," The Lancet Rheumatology, vol. 2, no. 10, pp. e603-e612, 2020.
[32] G. Guaraldi, M. Meschiari, A. Cozzi-Lepri et al., "Tocilizumab in patients with severe COVID-19: a retrospective cohort study," The Lancet Rheumatology, vol. 2, no. 8, pp. e474-e484, 2020.

[33] J. Radbel, N. Narayanan, and P. J. Bhatt, "Use of Tocilizumab for COVID-19-Induced Cytokine Release Syndrome: A Cautionary Case Report," Chest, vol. 158, no. 1, pp. e15-e19, 2020.

[34] R. Sanofi, Sanofi and Regeneron Provide Update on Kevzara ${ }^{\circledR}$ (Sarilumab) Phase 3 US Trial in COVID-19 Patients, Sanofi, 2020.

[35] S. G. Deftereos, G. Giannopoulos, D. A. Vrachatis et al., "Effect of colchicine vs standard care on cardiac and inflammatory biomarkers and clinical outcomes in patients hospitalized with coronavirus disease 2019: the GRECCO-19 randomized clinical trial," JAMA Network Open, vol. 3, no. 6, article e2013136, 2020.

[36] K. Kuba, Y. Imai, S. Rao et al., "A crucial role of angiotensin converting enzyme 2 (ACE2) in SARS coronavirus-induced lung injury," Nature Medicine, vol. 11, no. 8, pp. 875-879, 2005.

[37] D. E. Freedberg, J. Conigliaro, T. C. Wang et al., "Famotidine use is associated with improved clinical outcomes in hospitalized COVID-19 patients: a propensity score matched retrospective cohort study," Gastroenterology, vol. 159, no. 3, pp. 1129-1131.e3, 2020.

[38] T. Janowitz, E. Gablenz, D. Pattinson et al., "Famotidine use and quantitative symptom tracking for COVID-19 in nonhospitalised patients: a case series," Gut, vol. 69, no. 9, pp. 1592-1597, 2020.

[39] J. Wang, N. Hajizadeh, E. E. Moore et al., "Tissue plasminogen activator (tPA) treatment for COVID-19 associated acute respiratory distress syndrome (ARDS): a case series," Journal of Thrombosis and Haemostasis, vol. 18, no. 7, pp. 1752-1755, 2020.

[40] L.-A. Teuwen, V. Geldhof, A. Pasut, and P. Carmeliet, "Author Correction: COVID-19: the vasculature unleashed," Nature Reviews Immunology, vol. 20, no. 7, pp. 448-453, 2020.

[41] S. Chatterjee, "SGLT-2 inhibitors for COVID-19 - A miracle waiting to happen or just another beat around the bush?" Primary care diabetes, vol. 14, no. 5, pp. 564-565, 2020.

[42] V. Castiglione, M. Chiriacò, M. Emdin, S. Taddei, and G. Vergaro, "Statin therapy in COVID-19 infection," European Heart Journal-Cardiovascular Pharmacotherapy, vol. 6, no. 4, pp. 258-259, 2020.

[43] C. Mamoulakis, K. Tsarouhas, I. Fragkiadoulaki et al., "Contrast-induced nephropathy: basic concepts, pathophysiological implications and prevention strategies," Pharmacology \& therapeutics, vol. 180, pp. 99-112, 2017.

[44] B. Hudzik and J. Nowak, "Consideration of immunomodulatory actions of morphine in COVID-19-short report," European Review for Medical and Pharmacological Sciences, vol. 24, pp. 130262-130264, 2020.

[45] I. Rentoukas, G. Giannopoulos, A. Kaoukis et al., "Cardioprotective role of remote ischemic periconditioning in primary percutaneous coronary intervention: enhancement by opioid action," JACC. Cardiovascular Interventions, vol. 3, no. 1, pp. 49-55, 2010.

[46] Coronavirus Study Group of the International Committee on Taxonomy of Viruses, "The species Severe acute respiratory 
syndrome-related coronavirus: classifying 2019-nCoV and naming it SARS-CoV-2," Nature microbiology, vol. 5, no. 4, pp. 536-544, 2020.

[47] T. W. LeBaron, B. Kura, B. Kalocayova, N. Tribulova, and J. Slezak, "A new approach for the prevention and treatment of cardiovascular disorders. Molecular hydrogen significantly reduces the effects of oxidative stress," Molecules, vol. 24, no. 11, p. 2076, 2019.

[48] M. Yang, Y. Dong, Q. He et al., "Hydrogen: a novel option in human disease treatment," Oxidative Medicine and Cellular Longevity, vol. 2020, Article ID 8384742, 17 pages, 2020.

[49] E. Novo and M. Parola, "Redox mechanisms in hepatic chronic wound healing and fibrogenesis," Fibrogenesis \& Tissue Repair, vol. 1, no. 1, p. 5, 2008.

[50] I. Ohsawa, M. Ishikawa, K. Takahashi et al., "Hydrogen acts as a therapeutic antioxidant by selectively reducing cytotoxic oxygen radicals," Nature Medicine, vol. 13, no. 6, pp. 688694, 2007.

[51] S. Ohta, "Molecular hydrogen as a novel antioxidant: overview of the advantages of hydrogen for medical applications," Methods in enzymology, vol. 555, pp. 289-317, 2015.

[52] S. Ohta, "Molecular hydrogen as a preventive and therapeutic medical gas: initiation, development and potential of hydrogen medicine," Pharmacology \& Therapeutics, vol. 144, no. 1, pp. 1-11, 2014.

[53] M. Ichihara, S. Sobue, M. Ito, M. Ito, M. Hirayama, and K. Ohno, "Beneficial biological effects and the underlying mechanisms of molecular hydrogen-comprehensive review of 321 original articles," Medical Gas Research, vol. 5, no. 1, p. 12, 2015.

[54] T. G. Abisso, Y. M. Adzavon, P. Zhao, X. Zhang, M. Liu, and $\mathrm{X} . \mathrm{Ma}$, "Current progress in molecular hydrogen medication: protective and therapeutic uses of hydrogen against different disease scenarios," Internal Medicine, vol. 10, p. 314, 2020.

[55] W. Fang, L. Tang, G. Wang et al., "Molecular hydrogen protects human melanocytes from oxidative stress by activating Nrf2 signaling," The Journal of Investigative Dermatology, vol. 140, no. 11, pp. 2230-2241.e9, 2020.

[56] G. L. Nicolson, G. F. de Mattos, R. Settineri et al., "Clinical effects of hydrogen administration: from animal and human diseases to exercise medicine," International Journal of Clinical Medicine, vol. 7, no. 1, pp. 32-76, 2016.

[57] Y. Wu, M. Yuan, J. Song, X. Chen, and H. Yang, "Hydrogen gas from inflammation treatment to cancer therapy," ACS Nano, vol. 13, no. 8, pp. 8505-8511, 2019.

[58] C.-Y. Wu, W. L. Hsu, M. H. Tsai et al., "Hydrogen gas protects IP3Rs by reducing disulfide bridges in human keratinocytes under oxidative stress," Scientific Reports, vol. 7, no. 1, pp. 3606-3611, 2017.

[59] L. Li, X. Li, Z. Zhang, L. Liu, Y. Zhou, and F. Liu, "Protective mechanism and clinical application of hydrogen in myocardial ischemia-reperfusion injury," Pakistan Journal of Biological Sciences, vol. 23, no. 2, pp. 103-112, 2020.

[60] P. Qiu, Y. Liu, and J. Zhang, "Recent advances in studies of molecular hydrogen against sepsis," International Journal of Biological Sciences, vol. 15, no. 6, pp. 1261-1275, 2019.

[61] S. Ohta, "Recent progress toward hydrogen medicine: potential of molecular hydrogen for preventive and therapeutic applications," Current Pharmaceutical Design, vol. 17, no. 22, pp. 2241-2252, 2011.
[62] K. Xu, Hydrogen-Oxygen Inhalation for Treatment of COVID-19: With Commentary from Zhong Nanshan, World Scientific, 2020.

[63] Z. Nanshan, Use of mixed hydrogen and oxygen hydroxy gas inhalation for the treatment of COVID 19, 2020, https:// www.youtube.com/watch?v=42VgNLsZP0o.

[64] D. Alwazeer, "Potential cheap and effective drug for COVID19," 2020, https://www.researchgate.net/post/PotentialCheap-and-effective-Drug-for-COVID-19.

[65] G. Russell, M. Rehman, L. B. TW, D. Veal, E. Adukwu, and J. Hancock, "An overview of SARS-CoV-2 (COVID-19) infection and the importance of molecular hydrogen as an adjunctive therapy," Reactive Oxygen Species, vol. 10, pp. 150-165, 2020.

[66] T. W. LeBaron, M. L. McCullough, and K. H. Ruppman Sr., "A novel functional beverage for COVID-19 and other conditions: hypothesis and preliminary data, increased blood flow, and wound healing," Journal of Translational Science, vol. 6, pp. 1-6, 2020.

[67] K. Lucas, M. Rosch, and P. Langguth, "Molecular hydrogen (H2) as a potential treatment for acute and chronic fatigue," Archiv der Pharmazie (Weinheim), vol. 354, no. 4, article e2000378, 2021.

[68] M. Windholz, S. Budavari, L. Y. Stroumtsos, and M. N. Fertig, The Merck Index. An Encyclopedia of Chemicals and Drugs, vol. 354, no. 4, 1976Merck \& Co., 1976.

[69] Wired Chemist, Solubilities of gases in water at 293 K, 2020, http://www.wiredchemist.com/chemistry/data/solubilitiesgases.

[70] A. Shimouchi, K. Nose, M. Shirai, and T. Kondo, "Estimation of molecular hydrogen consumption in the human whole body after the ingestion of hydrogen-rich water," in Oxygen Transport to Tissue XXXIII, vol. 737, pp. 245-250, Springer New York, New York, NY, 2012.

[71] W. L. Jolly, Hydrogen, Encyclopædia Britannica, inc, 2020.

[72] Properties of Hydrogen, "Lumen," 2020, https://courses .lumenlearning.com/introchem/chapter/properties-ofhydrogen/.

[73] D. Alwazeer, K. Tan, and B. Örs, "Reducing atmosphere packaging as a novel alternative technique for extending shelf life of fresh cheese," Journal of Food Science and Technology, vol. 57, no. 8, article 4334, pp. 3013-3023, 2020.

[74] D. Alwazeer, "Importance of consideration of oxidoreduction potential as a critical quality parameter in food industries," Food Research International, vol. 132, article 109108, 2020.

[75] D. Alwazeer and B. Örs, "Reducing atmosphere drying as a novel drying technique for preserving the sensorial and nutritional notes of foods," Journal of food science and technology, vol. 56, no. 8, pp. 3790-3800, 2019.

[76] P. B. Hylemon, S. C. Harris, and J. M. Ridlon, "Metabolism of hydrogen gases and bile acids in the gut microbiome," FEBS Letters, vol. 592, no. 12, pp. 2070-2082, 2018.

[77] T. M. Olcott, Development of a Sorber Trace Contaminant Control System Including Pre- and Post-Sorbers for a Catalytic Oxidizer, National aeronautics and space administration Washington, DC, 1972.

[78] U. Hopfer, "Digestion and absorption of basic nutritional constituents," in Textbook of biochemistry with clinical correlation, pp. 1059-1091, Wiley-Liss New York, 1992.

[79] A. Shimouchi, K. Nose, M. Yamaguchi, H. Ishiguro, and T. Kondo, "Breath hydrogen produced by ingestion of 
commercial hydrogen water and milk," Biomarker Insights, vol. 4, 2009.

[80] Y. Muramatsu, M. Ito, T. Oshima, S. Kojima, and K. Ohno, "Hydrogen-rich water ameliorates bronchopulmonary dysplasia (BPD) in newborn rats," Pediatric Pulmonology, vol. 51, no. 9, pp. 928-935, 2016.

[81] K. Xie, Y. Yu, Y. Huang et al., "Molecular hydrogen ameliorates lipopolysaccharide-induced acute lung injury in mice through reducing inflammation and apoptosis," Shock, vol. 37, no. 5, pp. 548-555, 2012.

[82] Z.-Q. Zhou, C. H. Zhong, Z. Q. Su et al., "Breathing hydrogen-oxygen mixture decreases inspiratory effort in patients with tracheal stenosis," Respiration, vol. 97, no. 1, pp. 42-51, 2018.

[83] N. R. Council, Emergency and Continuous Exposure Guidance Levels for Selected Submarine Contaminants: Volume 3, vol. 3, National Academies Press, 2009.

[84] D. A. Crowl and Y. D. Jo, "The hazards and risks of hydrogen," Journal of Loss Prevention in the Process Industries, vol. 20, no. 2, pp. 158-164, 2007.

[85] S. H. Yousef, "Hydrogen safety: the road toward green technology," International Journal of Hydrogen Energy, vol. 38, no. 25, pp. 10716-10728, 2013.

[86] C. Tang, Z. Huang, C. Jin et al., "Explosion characteristics of hydrogen-nitrogen-air mixtures at elevated pressures and temperatures," International Journal of Hydrogen Energy, vol. 34, no. 1, pp. 554-561, 2009.

[87] S. Keten, C.-C. Chou, A. C. T. van Duin, and M. J. Buehler, "Tunable nanomechanics of protein disulfide bonds in redox microenvironments," Journal of the Mechanical Behavior of Biomedical Materials, vol. 5, no. 1, pp. 32-40, 2012.

[88] S. K. Biswas and I. Rahman, "Environmental toxicity, redox signaling and lung inflammation: the role of glutathione," Molecular Aspects of Medicine, vol. 30, no. 1-2, pp. 60-76, 2009.

[89] L. Mathys and J. Balzarini, "The role of cellular oxidoreductases in viral entry and virus infection-associated oxidative stress: potential therapeutic applications," Expert Opinion on Therapeutic Targets, vol. 20, no. 1, pp. 123-143, 2016.

[90] P. Checconi, M. de Angelis, M. E. Marcocci et al., "Redoxmodulating agents in the treatment of viral infections," International Journal of Molecular Sciences, vol. 21, no. 11, p. 4084, 2020.

[91] M. Roederer, S. W. Ela, F. J. T. Staal, L. A. Herzenberg, and L. A. Herzenberg, "N-Acetylcysteine: a new approach to anti-HIV therapy," AIDS Research and Human Retroviruses, vol. 8, no. 2, pp. 209-217, 1992.

[92] O. A. Khomich, S. N. Kochetkov, B. Bartosch, and A. V. Ivanov, "Redox biology of respiratory viral infections," Viruses, vol. 10, no. 8, p. 392, 2018.

[93] O. Van Hecke and J. Lee, N-Acetylcysteine: A Rapid Review of the Evidence for Effectiveness in Treating COVID-19, Nuffield Department of Primary Care Health Sciences, University of Oxford, 2020.

[94] O. Erel and S. Neselioglu, "A novel and automated assay for thiol/disulphide homeostasis," Clinical Biochemistry, vol. 47, no. 18 , pp. 326-332, 2014.

[95] D. Petrakis, D. Margină, K. Tsarouhas et al., "Obesity - a risk factor for increased COVID-19 prevalence, severity and lethality (Review)," Molecular Medicine Reports, vol. 22, no. 1, pp. 9-19, 2020.
[96] H. Zhou, B. Han, L. M. Hou et al., "Protective effects of hydrogen gas on experimental acute pancreatitis," PLoS One, vol. 11, no. 4, article e0154483, 2016.

[97] B. Kura, A. K. Bagchi, P. K. Singal et al., "Molecular hydrogen: potential in mitigating oxidative-stress-induced radiation injury," Canadian Journal of Physiology and Pharmacology, vol. 97, no. 4, pp. 287-292, 2019.

[98] H.-T. Yao, Y.-H. Yang, and M.-L. Li, "Intake of molecular hydrogen in drinking water increases membrane transporters, p-glycoprotein, and multidrug resistance-associated protein 2 without affecting xenobiotic-metabolizing enzymes in rat liver," Molecules, vol. 24, no. 14, p. 2627, 2019.

[99] W. Liu, L. P. Shan, X. S. Dong, X. W. Liu, T. Ma, and Z. Liu, "Combined early fluid resuscitation and hydrogen inhalation attenuates lung and intestine injury," World journal of gastroenterology: WJG, vol. 19, no. 4, pp. 492-502, 2013.

[100] A. Nakao, Y. Toyoda, P. Sharma, M. Evans, and N. Guthrie, "Effectiveness of hydrogen rich water on antioxidant status of subjects with potential metabolic syndrome-an open label pilot study," Journal of Clinical Biochemistry and Nutrition, vol. 46, no. 2, pp. 140-149, 2010.

[101] Y.-S. Zhao, J. R. An, S. Yang et al., "Hydrogen and oxygen mixture to improve cardiac dysfunction and myocardial pathological changes induced by intermittent hypoxia in rats," Oxidative Medicine and Cellular Longevity, vol. 2019, Article ID 7415212, 12 pages, 2019.

[102] Y. Suzuki, T. Sato, M. Sugimoto et al., "Hydrogen-rich pure water prevents cigarette smoke-induced pulmonary emphysema in SMP30 knockout mice," Biochemical and Biophysical Research Communications, vol. 492, no. 1, pp. 74-81, 2017.

[103] C. Jieun, S. Eun, B. Young-Hwan et al., "Hydrogen-enriched water eliminates fine particles from the lungs and blood by enhancing phagocytic activity," Journal of Biomedical Research, vol. 31, no. 6, p. 503, 2017.

[104] B. A. S. E. M. Task Force, "COVID-19: facts and recommendations from A to Z," Sci Insigt, vol. 33, no. 1, pp. 138-158, 2020.

[105] K. Nagatani, K. Wada, S. Takeuchi et al., "Effect of hydrogen gas on the survival rate of mice following global cerebral ischemia," Shock, vol. 37, no. 6, pp. 645-652, 2012.

[106] R. K. L. Bouhafs and C. Jarstrand, "Effects of antioxidants on surfactant peroxidation by stimulated human polymorphonuclear leukocytes," Free Radical Research, vol. 36, no. 7, pp. 727-734, 2002.

[107] C. E. Cross, A. Van der Vliet, S. Louie, J. J. Thiele, and B. Halliwell, "Oxidative stress and antioxidants at biosurfaces: plants, skin, and respiratory tract surfaces," Environmental Health Perspectives, vol. 106, supplement 5, pp. 1241-1251, 1998.

[108] C. Dani and C. Poggi, "Antioxidant properties of surfactant," in Perinatal and Prenatal Disorders, pp. 245-254, Springer, 2014.

[109] K. Rodríguez-Capote, D. Manzanares, T. Haines, and F. Possmayer, "Reactive oxygen species inactivation of surfactant involves structural and functional alterations to surfactant proteins SP-B and SP-C," Biophysical Journal, vol. 90, no. 8, pp. 2808-2821, 2006.

[110] S. Andersson, A. Kheiter, and T. A. Merritt, "Oxidative inactivation of surfactants," Lung, vol. 177, no. 3, pp. 179-189, 1999. 
[111] K. Kurashima, N. Kagiyama, T. Ishiguro et al., "Risk factors for the severe COVID-19 pneumonia," The Japanese Association for Infectious Disease, pp. 1-7, 2020.

[112] K. Sriram and P. A. Insel, "A hypothesis for pathobiology and treatment of COVID-19: the centrality of ACE1/ACE2 imbalance," British Journal of Pharmacology, vol. 177, no. 21, pp. 4825-4844, 2020.

[113] F. Farooqi, N. Dhawan, R. Morgan, J. Dinh, K. Nedd, and G. Yatzkan, "Treatment of severe COVID-19 with tocilizumab mitigates cytokine storm and averts mechanical ventilation during acute respiratory distress: a case report and literature review," Tropical medicine and infectious disease, vol. 5 , no. 3 , p. $112,2020$.

[114] T. W. LeBaron, I. Laher, B. Kura, and J. Slezak, "Hydrogen gas: from clinical medicine to an emerging ergogenic molecule for sports athletes," Canadian Journal of Physiology and Pharmacology, vol. 97, no. 9, pp. 797-807, 2019.

[115] N. Zhong, Fighting against COVID-19 Using Hydrogen-Oxygen Therapy, 2020, https://www.youtube.com/watch?v= W8MZuB8KuCE.

[116] J. K. Aronson, R. E. Ferner, N. DeVito, and C. Heneghan, COVID-19 Trials Registered up to 8 March 2020-An Analysis of 382 Studies, The Centre for Evidence-Based Medicine, Oxford, 2020.

[117] T. C. Center, “Clinical test registration,” 2021, https://www .chictr.org.cn/searchprojen.aspx?title=hydrogen\&officialname $=$ covid-19\&subjectid=\&secondaryid $=$ \&applier $=\&$ studyleader $=$ \&ethicalcommitteesanction $=\&$ sponsor $=\&$ studyailment $=$ $\&$ studyailmentcode $=\&$ studytype $=0 \&$ studystage $=$ $0 \&$ studydesign $=0 \&$ minstudyexecutetime $=\&$ ma .

[118] US National Library of Medecine, "ClinicalTrials.gov US National Library of Medicine2021," https://clinicaltrials .gov/ct $2 /$ results? cond=Covid-19\&term=hydrogen \&cntry= \&state $=\&$ city $=\&$ dist.

[119] S. Westaby, "From Wuhan to Westminster: can NHS critical care cope with coronavirus?," The Bulletin of the Royal College of Surgeons of England, vol. 102, no. 4, pp. 120-125, 2020. 